\title{
L'abbaye Saint-Michel de Cuxa à Codalet (Pyrénées- Orientales), nouvelles données archéologiques sur les origines
}

\section{Fabrice Henrion}

\author{
(2) OpenEdition \\ 12 Journals

\section{Édition électronique} \\ URL : https://journals.openedition.org/cem/14677 \\ DOI : $10.4000 /$ cem. 14677 \\ ISSN : 1954-3093 \\ Éditeur \\ Centre d'études médiévales Saint-Germain d'Auxerre
}

Référence électronique

Fabrice Henrion, «L'abbaye Saint-Michel de Cuxa à Codalet (Pyrénées-Orientales), nouvelles données archéologiques sur les origines », Bulletin du centre d'études médiévales d'Auxerre / BUCEMA [En ligne], 21.1 | 2017, mis en ligne le 18 septembre 2017, consulté le 22 septembre 2022. URL : http:// journals.openedition.org/cem/14677 ; DOI : https://doi.org/10.4000/cem.14677

Ce document a été généré automatiquement le 22 septembre 2022.

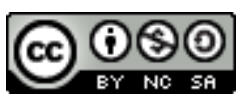

Creative Commons - Attribution - Pas d'Utilisation Commerciale - Partage dans les Mêmes Conditions 4.0 International - CC BY-NC-SA 4.0

https://creativecommons.org/licenses/by-nc-sa/4.0/ 


\title{
L'abbaye Saint-Michel de Cuxa à Codalet (Pyrénées-Orientales), nouvelles données archéologiques sur les origines
}

\author{
Fabrice Henrion
}

1 Située sur le piémont nord du massif du Canigou à $5 \mathrm{~km}$ au sud de Prades, au sud de la partie occidentale de la vallée de la Têt - région naturelle du Bas-Conflent -, l'abbaye Saint-Michel de Cuxa a été implantée dans la partie inférieure d'un replat formé par un cône de déjection alimenté par un torrent de la rive méridionale de la Têt (la Llitera). Ce cône de moyenne terrasse, orienté nord-sud, est constitué de cailloutis à gros éléments peu roulés (schistes, calcaires, etc.) provenant des terrains amonts (fig. 1).

2 Les terrains géologiques sous-jacents et latéraux à ce cône de déjection appartiennent essentiellement à la Formation de Codalet du Burdigalien inférieur. Bien visible dans le lit de la Llitera entre Taurinya et Prades, elle est constituée d'éléments empruntés à la série de Carnaveilles (micaschites, quartz, calcaires) emballés dans une matrice argileuse rougeâtre. Les éléments sont de petite taille et le plus souvent anguleux. 
Fig. 1 - Vue générale de l'abbaye depuis l'ouest

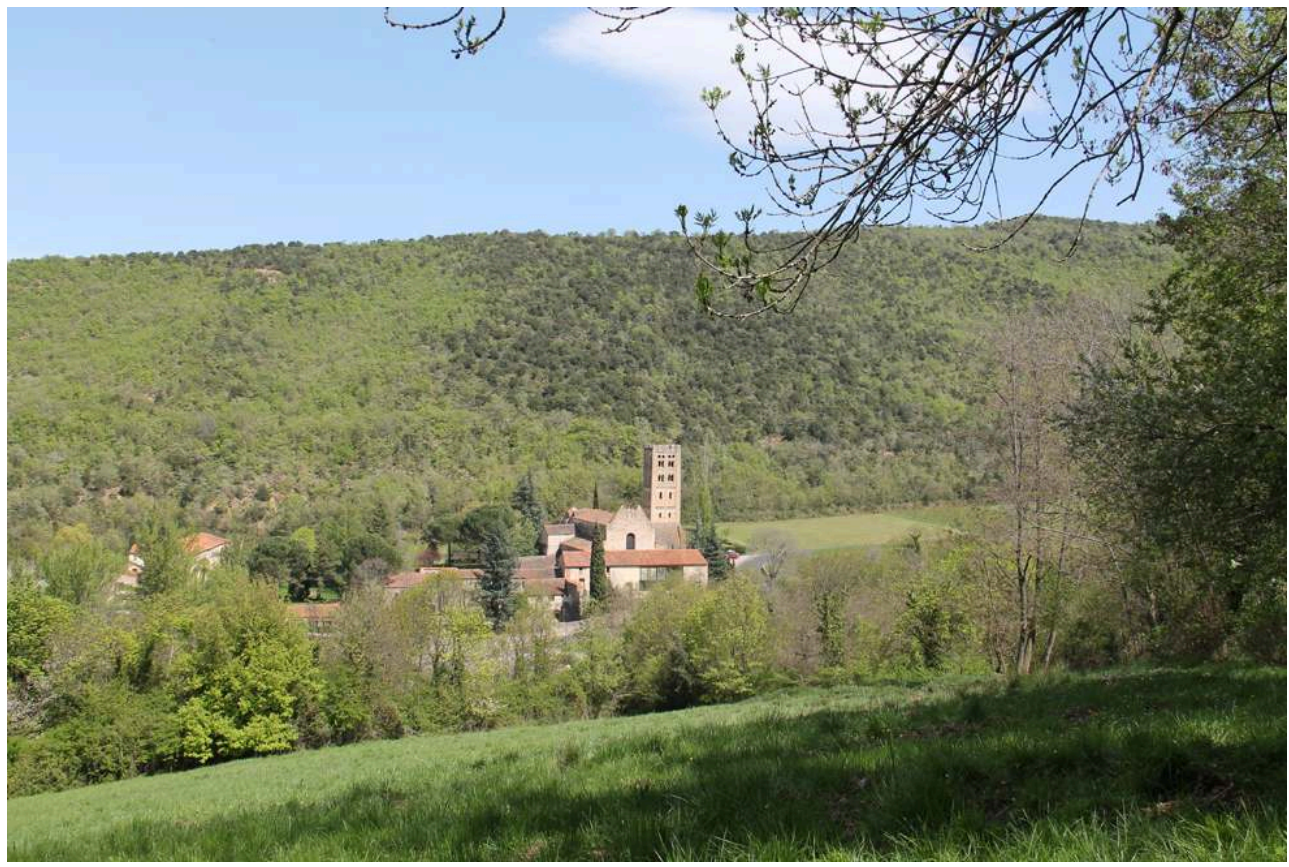

CEM 2016

3 Depuis au moins le milieu du $\mathrm{IX}^{\mathrm{e}}$ siècle existe à Cuxa une petite communauté de moines, rassemblée autour de Protasius, et peut-être déjà organisée avec une église dédiée à saint Germain d'Auxerre' ${ }^{1}$. La communauté de Cuxa se rapproche en 864-865 de celle de Saint-André d'Eixalada, établie depuis les années 840 en haut Conflent. Leur destin commun s'affirme de nouveau à l'automne 878 , lorsqu'une crue gigantesque de la rivière Têt emporte les bâtiments du monastère Saint-André et, avec eux, une grande partie de la communauté. Les survivants, dont l'abbé Baron, quittent alors définitivement Eixalada pour s'installer à Cuxa et Protasius prend la tête de la nouvelle communauté, qui devient l'abbaye Saint-Germain de Cuxa (19 juin 879) ${ }^{2}$. Les bâtiments sont alors agrandis grâce à Miron, comte de Conflent, à l'instar du domaine et des possessions. Dans son testament (13 septembre 879$)^{3}$, Protasius confie à la protection du comte Miron l'avenir de ses cinquante moines, vingt servants et de l'ensemble des biens et lui demande d'encourager le développement du monastère, comme il l'a fait par le passé. Les travaux d'agrandissement des bâtiments de Cuxa ont pu commencer dès l'abandon d'Eixalada, en tout cas ils semblent achevés à la fin de l'été 879 , comme le suggérerait une allusion dans le testament de Protasius ${ }^{4}$.

4 Un long silence suit ce renouveau du monastère et il faut attendre l'année 938 pour qu'une première mention rende compte de la présence d'une seconde église, dédiée à saint Michel, pour laquelle un document postérieur indique qu'elle était petite et circulaire. L'église Saint-Germain, jusqu'alors " de terre et de pierres », est ensuite reconstruite " avec de la chaux, des pierres et des bois travaillés merveilleusement » sous l'abbatiat de Gondefred et l'impulsion du comte Siniofred ; elle est consacrée le 30 juillet 953. L'acte de consécration précise qu'elle constituait le chevet de l'abbatiale dédiée à saint Germain et à saint Michel, qui fait également l'objet de reconstruction quelques années plus tard sous l'abbatiat de Pons, pour être consacrée le 28 septembre 975 sous l'abbé Garin5. 
L'ensemble est encore agrandi sous l'abbé Oliba (1008-1046) avec, en particulier, les constructions occidentales, crypte, chapelle supérieure de la Trinité et inférieure de la Crèche et atrium. On ne sait quasiment rien des bâtiments monastiques ni du cloître au cours de ces périodes hautes (fig. 2).

Fig. 2 - Plan général de l'abbaye dans son état actuel, avec indication des espaces cités

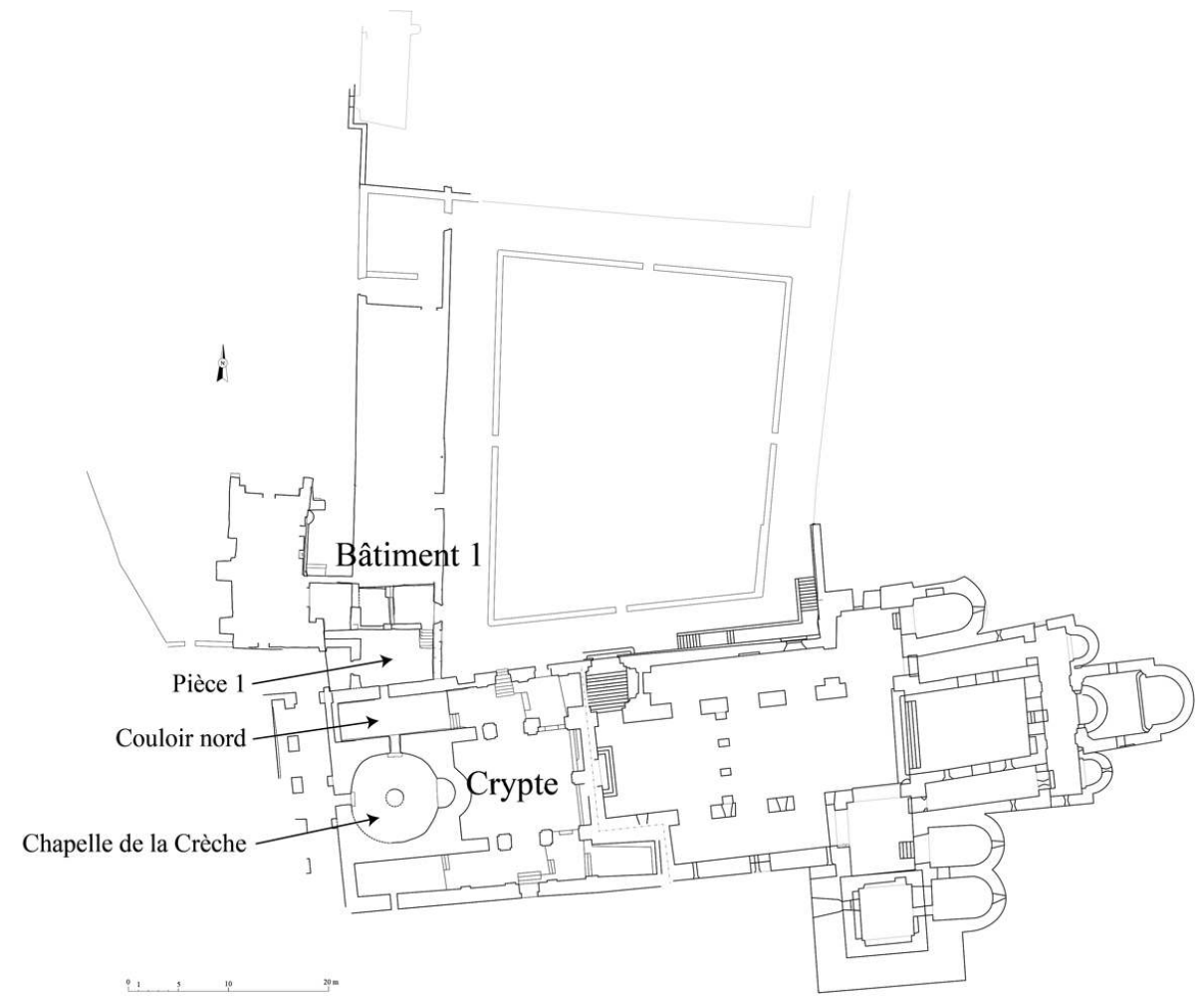

Plan J.-L. Bernard 1993 et CEM 2016

Une première intervention archéologique, associant sondages au sol et étude de bâti, menée en 1999 sous la direction de Philippe Alessandri et Astrid Huser (AFAN), entre autres sur le logis du Grand-Sacristain (bâtiment 1), qui prend place à l'extrémité méridionale de l'aile occidentale du cloître du XII ${ }^{e}$ siècle, avait permis de montrer que les dispositions romanes reprenaient ici une construction plus ancienne, datable du $\mathrm{x}^{\mathrm{e}}$ siècle par la typologie des ouvertures (arcs outrepassés). Par ailleurs, la mise au jour de deux inhumations en coffrage de pierres montées à l'argile (sondage 7) antérieures à ce bâtiment devait relancer la réflexion sur la question des origines du monastère et de l'occupation du site avant la seconde moitié du $\mathrm{IX}^{\mathrm{e}}$ siècle. Seule la tombe au sud (sépulture 1) avait alors été fouillée et la tombe au nord (sépulture 2) n'a pu être étudiée qu'en 2016 ; la sépulture 1 accueillait l'inhumation d'un individu adulte de sexe féminin avec son fœetus. Un second sondage avait été ouvert plus au sud (sondage 5), au pied de la porte menant à la crypte. La stratigraphie reconnue témoignait d'une certaine complexité, non pas dans la succession des événements somme toute simple, mais plutôt dans sa relation avec l'ensemble de l'espace et l'incohérence altimétrique des occupations entre elles ${ }^{6}$.

7 La fouille programmée menée au printemps 2016 avait pour objectifs d'étudier en aire ouverte l'ensemble des sols conservés dans cette partie de l'aile occidentale des bâtiments claustraux et d'affiner l'étude de bâti en mettant en œuvre toutes les 
méthodes susceptibles d'apporter les informations nécessaires à la compréhension du site : analyse des matériaux mis en œuvre (pierres et mortiers), datations radiocarbone, etc. La crypte a également fait l'objet d'une attention particulière et, plus spécialement, son couloir nord qui côtoie le bâtiment 1 . Les résultats obtenus modifient considérablement la perception tant des origines du site que de son évolution au cours des premiers siècles de son histoire et justifient pleinement une poursuite des recherches.

Bien que les niveaux d'occupation les plus anciens aient en grande partie disparu avec les travaux ultérieurs, on peut proposer, à partir des données acquises et de leur analyse raisonnée de manière régressive, l'état le plus ancien sur lequel viendront s'installer les deux inhumations fouillées. La reconnaissance de la position du terrain naturel pédogénéisé permet d'envisager la configuration des lieux avec une forte pente d'est en ouest, suivant la topographie naturelle, descendant vers le cours de la Llitera, sans toutefois pouvoir déterminer le niveau de circulation.

\section{Une première occupation funéraire et bâtie (VIII ${ }^{\mathrm{e}}-\mathrm{IX}$ siècle ?)}

9 C'est dans le haut de cette pente et, sans doute, à la rupture de celle-ci, que seront installées les inhumations en coffrage de pierres, peut-être dans la partie occidentale d'un premier bâtiment en bois se développant à l'est et, sans doute, au nord et au sud (fig. 3).

Fig. 3 - Proposition de plan pour l'état 1 du bâtiment 1 (fin VIII -IX ${ }^{e}$ siècle), hypothèses 2016

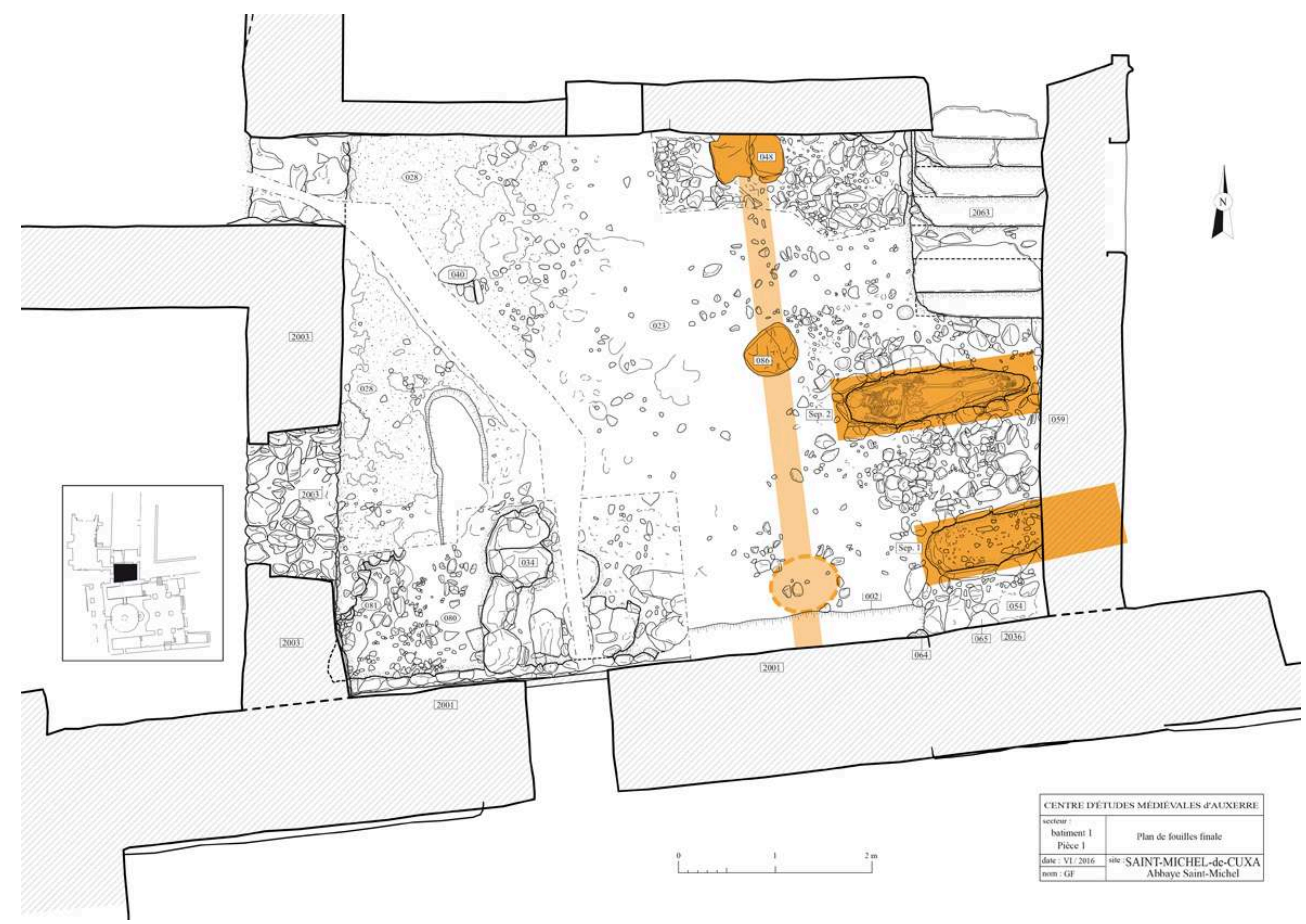

CEM 2016

Les indices sont ténus pour l'envisager concrètement, mais l'alignement des supports, ou de leur souvenir, s'accorde avec les orientations des dispositions plus tardives, 
comme l'église, et la crypte pour laquelle on pressent au moins un état antérieur. Il est encore trop tôt pour en restituer le plan, ni même supposer une relation avec des constructions plus au sud que seule une fouille, au moins du couloir nord de la crypte, pourrait illustrer, mais tout porte à croire qu'une occupation antérieure, ou à peine, à l'arrivée de la communauté d'Eixalada a bien existé.

11 Nous n'avons pas d'indice de datation, autre que typologique et stratigraphique, pour la sépulture 1, celle de la femme avec son fœtus. Son coffrage de dalles posées de chant, montées à l'argile, formant un plan légèrement ovoïdal, se rapproche d'exemplaires de Saint-Martin de Castries (Hérault), datés entre la fin du viII siècle et le dernier tiers du $\mathrm{IX}^{\mathrm{e}}$ siècle $^{7}$, et son antériorité à la construction du bâtiment 1 au $\mathrm{X}^{\mathrm{e}}$ siècle est stratigraphiquement avérée. D'un point de vue chronologique, elle ne doit pas être très éloignée de la sépulture 2 (un homme adulte), malgré les différences typologiques. Le plan ovoïdal bien marqué de son coffrage de pierres montées à l'argile renvoie par comparaisons à des exemplaires datés entre la fin $d u \mathrm{VII}^{\mathrm{e}}$ et le $\mathrm{IX}^{\mathrm{e}}$ siècle ${ }^{8}$, mais on retiendra également la datation radiocarbone d'un échantillon osseux qui situerait l'inhumation entre la fin du $\mathrm{IX}^{\mathrm{e}}$ et le $\mathrm{X}^{\mathrm{e}}$ siècle ${ }^{9}$. On a tendance, sans doute par facilité, à associer les deux tombes dans un même espace, voire dans un même temps, sans que l'on puisse en mesurer l'épaisseur, mais rien n'empêche que la sépulture 2 ait été mise en place non pas dans le probable bâtiment en bois du premier état, mais dans le bâtiment 1 , à proximité de la sépulture 1 , dont la présence était connue ou reconnue ${ }^{10}$. Cette question ne peut malheureusement plus trouver de réponses matérielles tant les décaissements romans ont pu être radicaux, mais l'analyse des phases postérieures peut apporter un certain éclairage. La question de la présence de ces inhumations ne peut pas non plus être résolue, à l'instar de celle concernant la fonction de l'éventuel premier bâtiment en bois. Si l'on considère les datations proposées, cette première occupation décelée prendrait place dans la période voyant l'arrivée de la communauté d'Eixalada et la réorganisation des lieux grâce au comte Miron, à moins qu'elle ne renvoie à ce qui a précédé la fondation monastique. Mais cela ne donne pas de fonction au premier bâtiment, d'autant qu'on ne connaît pas l'emplacement de l'église d'alors, ni du cloître, s'il y en a un. Et le fait qu'on ne connaisse pas non plus la fonction précise de ce premier bâtiment ne permet pas de raisonner sur le maintien des usages au même endroit. Toujours est-il qu'il accueille, selon toute vraisemblance, la ou les tombes, dont le caractère privilégié peut être retenu, et qu'il paraît difficile alors de lui attribuer un rôle strictement utilitaire.

\section{Construction et fonction du bâtiment 1 au $x^{\mathrm{e}}$ siècle}

La datation radiocarbone d'échantillons de charbons de bois prélevés dans les mortiers de mise en œuvre du mur est du bâtiment 1 place sa construction entre la fin du $\mathrm{IX}^{\mathrm{e}}$ siècle et la première moitié $\mathrm{du} \mathrm{X}^{\mathrm{e}}$ siècle $^{11}$. La cohérence typologique des mortiers nous assure que son mur ouest appartient à la même phase de chantier; le mobilier recueilli dans les niveaux de construction s'accorde également avec cette datation. On est donc en présence d'un bâtiment homogène, orienté nord-sud, d'un peu plus de $7 \mathrm{~m}$ de largeur, dont l'extension vers le nord n'est pas connue. Au sud, les indices perçus dans la crypte montrent que son pignon est repris par le mur nord du couloir, dont des lambeaux sont préservés à l'est d'un bouchage. Ces vestiges conservent, par ailleurs, la trace d'un piédroit correspondant à un arcosolium plutôt qu'à une ouverture, car elle 
ouvrirait sinon sur le remblai d'exhaussement des sols du bâtiment. Il n'en demeure pas moins que l'arcosolium s'enfonce un peu en forme de niche dans ce remblai et le muret bahut se prolonge dans la niche par un aménagement de pierres à plat, scellées à l'argile, bloqué contre le coffrage de la sépulture 1. Il s'agit manifestement d'une monumentalisation et un maintien de la mémoire des tombes situées derrière, que l'exhaussement des sols masque désormais aux yeux des vivants. On ne sait pas si cet aménagement sur le pignon est dans un espace bâti, dont on n'a aucune trace, ou à l'extérieur. Toutefois, le pignon ainsi restitué n'est pas perpendiculaire aux gouttereaux et cette configuration ne semble pas pouvoir être le résultat d'une contrainte topographique, à l'inverse de l'orientation du bâtiment, comme on l'a vu. Ce biais du pignon peut, par contre, refléter la présence au sud d'une construction antérieure mais encore conservée dans cette première moitié $d u x^{e}$ siècle, à laquelle succédera la crypte quelques générations plus tard.

Les sols d'occupation du bâtiment ont été détruits par les réaménagements $\mathrm{du} \mathrm{XI}^{\mathrm{e}}$ siècle, mais on peut en restituer la position à partir du seuil de la porte dans le mur est. Ils résultent d'un remblaiement à la suite de la construction du bâtiment 1 , dont le chantier s'implante dans la pente naturelle, expliquant ainsi la différence altimétrique entre la fondation est et la fondation ouest (fig. 4).

Fig. 4 a - Proposition de restitution du profil de la topographie naturelle et des sols du bâtiment 1

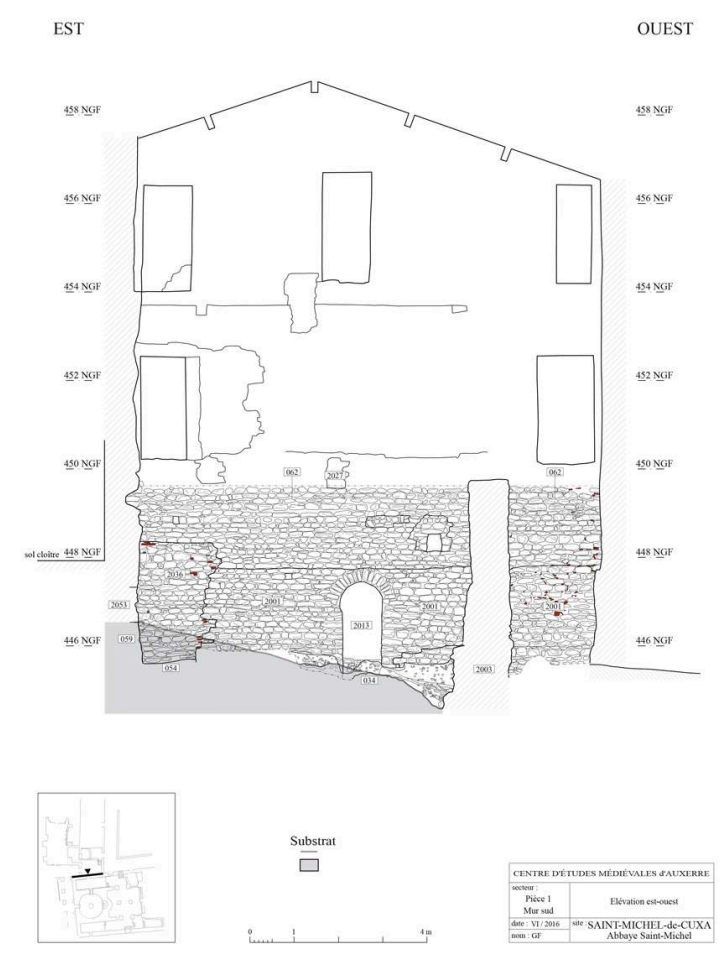


Fig. 4 b - Proposition de restitution du profil de la topographie naturelle et des sols de l'état 1 (fin $\mathrm{VIII}^{\mathrm{e}}-\mathrm{IX} \mathrm{e}$ siècle) du bâtiment 1

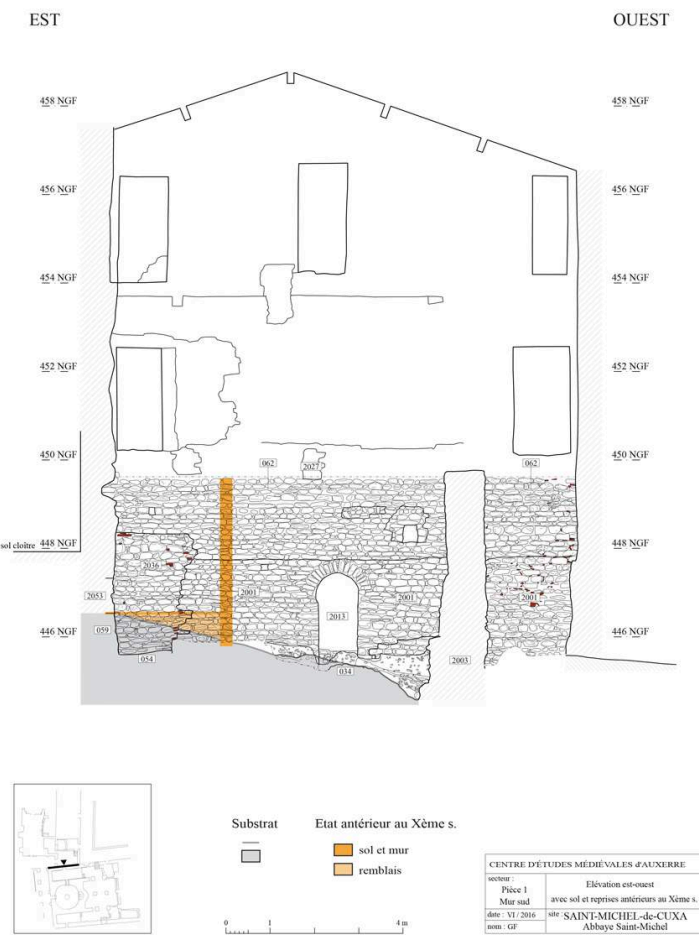

CEM 2016 
Fig. $4 \mathrm{c}$ - Proposition de restitution du profil de la topographie naturelle et des sols de l'état 2 ( $\mathrm{x}^{\mathrm{e}}$ siècle) du bâtiment 1

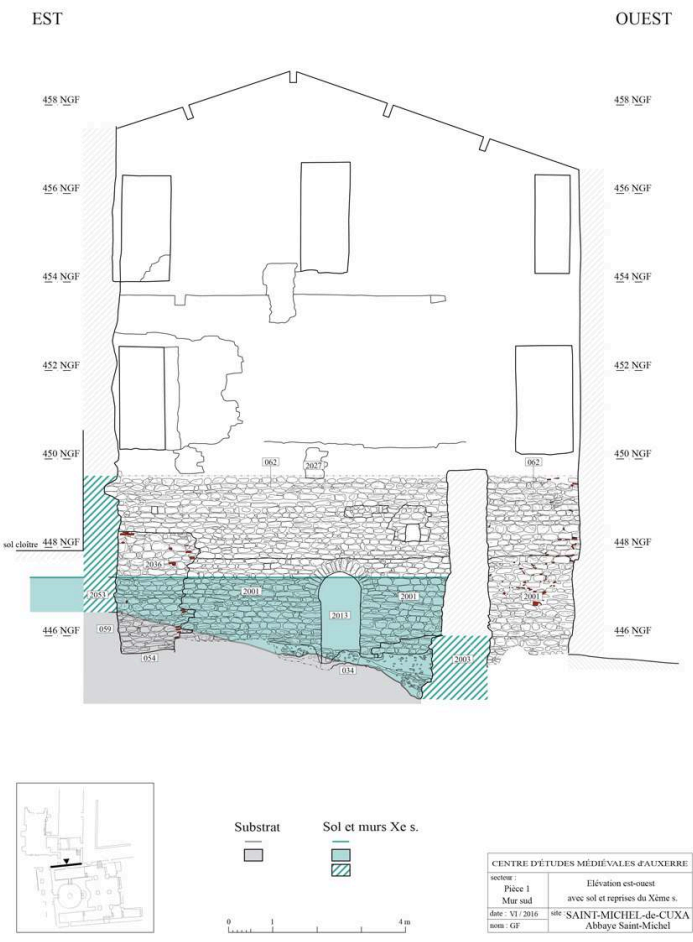

CEM 2016 
Fig. $4 \mathrm{~d}$ - Proposition de restitution du profil de la topographie naturelle et des sols de l'état 3 ( $\mathrm{xl}$ e siècle) du bâtiment 1

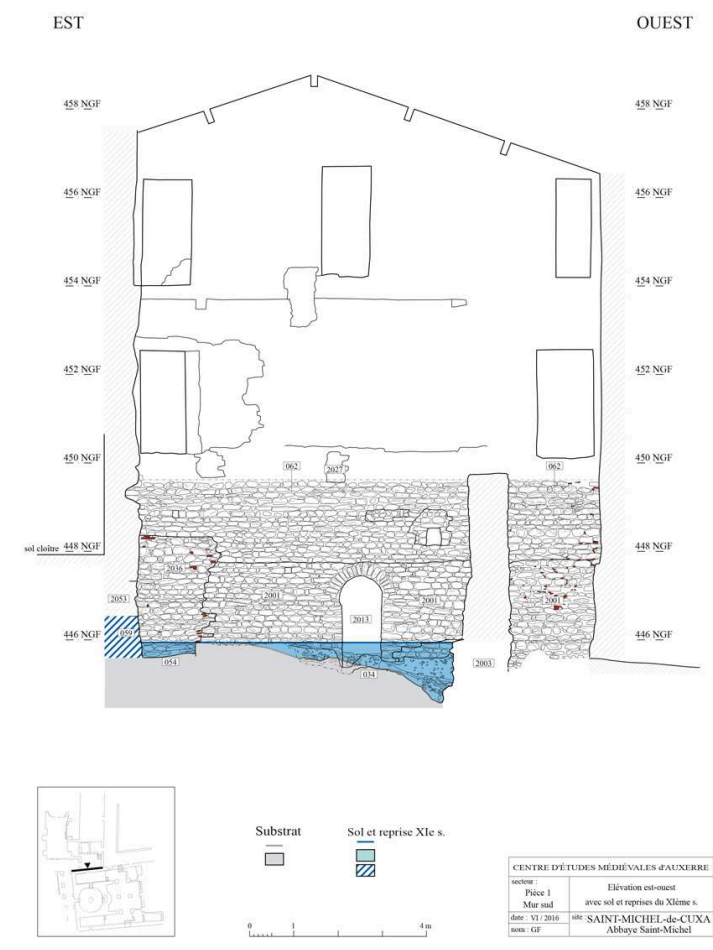

CEM 2016 du bâtiment d'accueil. Plutôt qu’à des supports de plafonnement d'une pièce, on peut penser qu'elles reçoivent la structure d'une galerie haute desservant le bâtiment, dont le sol d'occupation est plus haut compte tenu des remblaiements, sans que l'on sache si on emprunte cette galerie depuis le nord ou le sud. La très forte altération des enduits de la pile sud, uniquement à l'ouest, s'expliquerait par son exposition aux intempéries, alors que le côté oriental est protégé. La typologie des mortiers ne permet pas d'associer la construction des piles au chantier de la première moitié du $x^{e}$ siècle, mais il peut s'agir d'un second chantier, toujours dans le même siècle.

Finalement, aucun élément ne permet d'envisager la fonction de ce bâtiment, sans doute déjà accolé à une autre construction au sud, mais séparé de l'emprise du cloître telle que proposée actuellement. L'idée avancée d'un bâtiment d'accueil ne doit pas être abandonnée, d'autant moins si l'on retient l'hypothèse de la galerie extérieure, comme à Cluny pour les Hostelleries de Saint-Hugues, ou celle suggérant que l'on soit en présence du logis de l'abbé. Mais la datation précoce donnée par le radiocarbone

permet également d'envisager un premier bâtiment ${ }^{12}$ destiné à accueillir une

apport massif de terres ne vienne rétablir les niveaux. Il est possible également
a dentement du chantier a progressivement comblé la pente l'ouest, avant qun d'envisager que la position des sols intérieurs s'accorde avec les niveaux de circulation extérieurs, à l'est, alors qu'à l'ouest la pente naturelle est maintenue.

On ne connaît que les portes orientales et l'arase du gouttereau occidental est trop basse pour l'on puisse déceler des indices d'ouvertures, mais on peut penser qu'aucune n'existait de ce côté, le seul a priori contreforté.

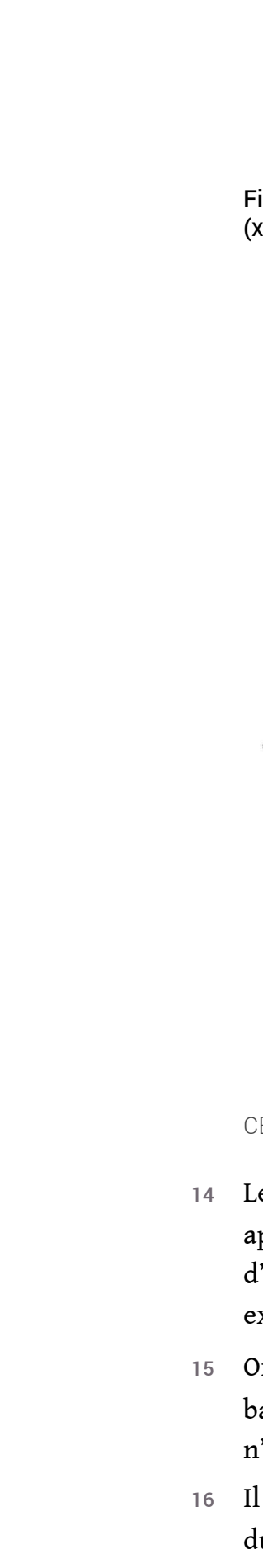


communauté devenant plus nombreuse avec l'arrivée des moines d'Eixalada, avant qu'un programme de constructions idoines ne se développe (fig. 5).

Fig. 5 - Proposition de restitution du plan de l'état 2 du monastère ( $x^{e}$ siècle), hypothèses 2016

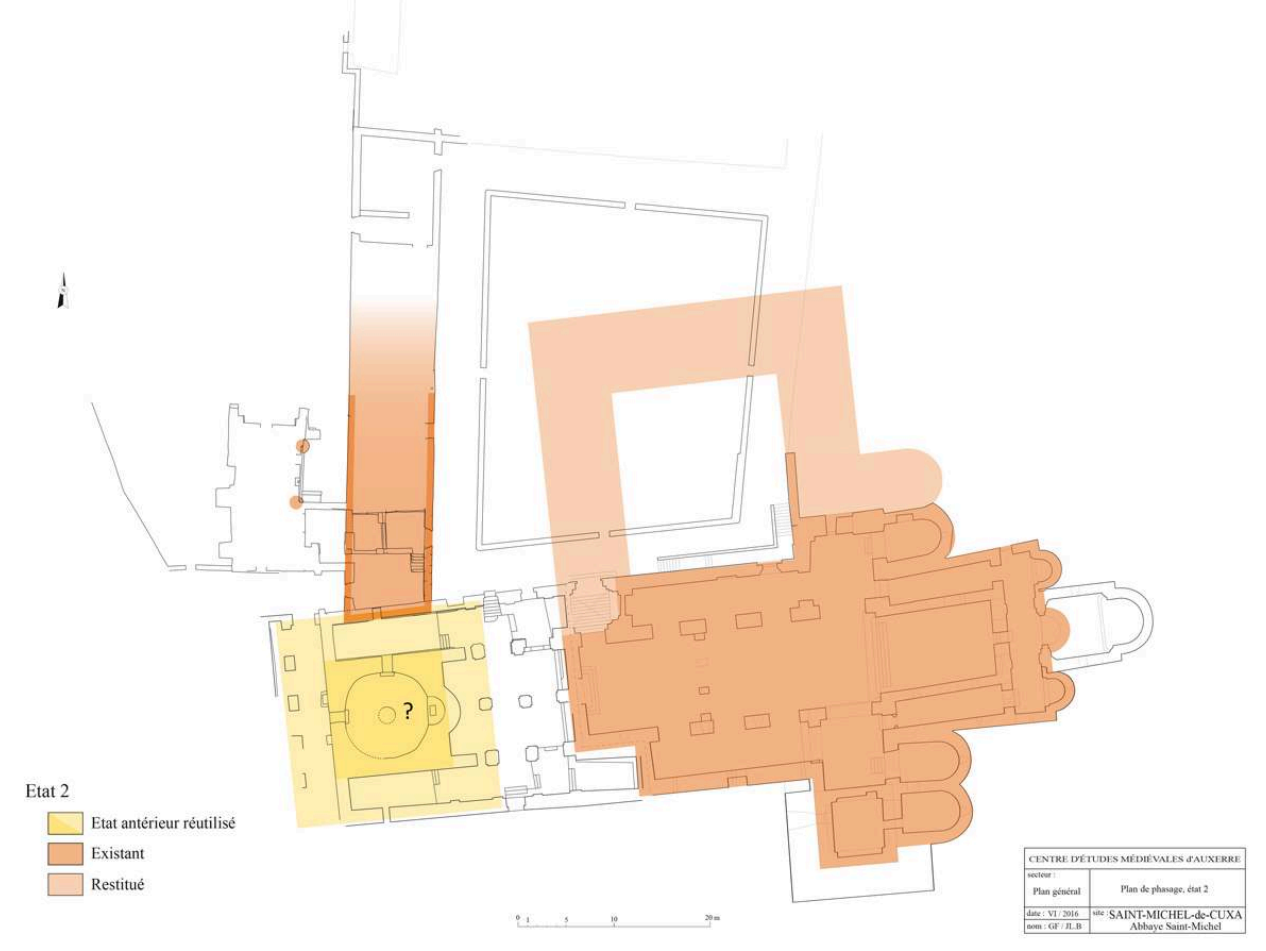

\section{Le chantier du $\mathrm{XI}^{\mathrm{e}}$ siècle}

Si l'on se réfère aux données historiographiques disponibles, le chantier du $\mathrm{xI}^{\mathrm{e}}$ siècle concernerait en particulier la construction de la crypte, de la chapelle de la Trinité qui la surmonte et de l'atrium, mais les données nouvellement acquises renvoient une image beaucoup plus complexe. Il était admis que l'élévation sud de la pièce $1 \mathrm{du}$ bâtiment 1 résultait d'une même phase de construction que la crypte et la chapelle de la Trinité, mais on a vu que l'analyse plus fine de la maçonnerie permettait de comprendre plusieurs étapes sans doute obligées par les dispositions en place.

Dans l'espace de la pièce 1 , l'engagement des travaux concerne d'abord un rabaissement des sols jusqu'au-dessus des deux inhumations qui seront préservées. Ce décaissement descendant plus bas que la base de la fondation du mur oriental oblige à le reprendre en sous-œuvre. Puis, dans un second temps, la tranchée de construction du mur sud est creusée, en s'interrompant à l'est aux abords de l'aménagement de l'arcosolium qui sera ensuite repris. Une brèche est laissée dans le mur sud, sans doute pour la circulation durant le chantier. Tout porte à croire que le pignon de l'état antérieur est d'abord chemisé, en particulier son parement nord, avant d'être supprimé par la construction du mur sud.

La brèche sera finalement bouchée et la porte d'accès à la crypte sera créée. 
21 La différence d'altitude entre le sol de la pièce 1 et celui de la crypte sera compensée par un emmarchement, dont le massif de fondation s'appuie sur la semelle du mur sud et sur le seuil de la porte, témoignant qu'il intervient bien dans une ultime étape d'aménagement.

La modification de la hauteur du sol s'accompagne également d'un changement dans les circulations et les accès au bâtiment puisque la porte orientale est bouchée au même moment.

L'arcosolium, un temps réaménagé, sera finalement bouché en même temps que la création de l'emmarchement d'accès à la crypte, comme en témoigne la similitude des mortiers de mise en œuvre.

On peut restituer la hauteur sous plafond à un peu plus de $4 \mathrm{~m}$ déterminée à partir des réservations de poutres préservées dans le mur sud, ce qui confère à cet espace un volume important, sans que l'on puisse lui attribuer de fonction. Toutefois, la présence d'une porte d'accès à la crypte est un indice particulièrement important, qui permet d'exclure d'emblée une fonction de cellier ou de bâtiment de stockage, comme sa position à l'ouest pourrait le laisser penser. En revanche, que ce bâtiment puisse être le logis de l'abbé justifierait l'organisation des circulations - suppression de l'accès vers l'est et création d'un accès direct à la crypte - et l'importance des volumes digne d'une grande salle.

25 Dans le même temps, des constructions sont accolées à l'ouest du bâtiment, sans que l'on puisse déterminer si la galerie est alors supprimée ou simplement modifiée. L'ajout de constructions peut aussi participer à son renforcement, justifié par le fait qu'elle est construite dans la pente.

\section{La crypte}

La complexité du mur nord du couloir nord de la crypte témoigne d'une construction par étapes suivant les contraintes dues à la présence du bâtiment 1 et de son pignon sud. La même complexité dans les étapes ou les phases de construction dans le reste de la crypte est également perceptible d'après l'étude des mortiers et les quelques observations de bâti qui ont pu être réalisées. Si on retrouve globalement les mêmes typologies de mortier entre le couloir nord et le bâtiment 1, quels que soient les états considérés, la typologie du mortier mis en œuvre pour la construction de la chapelle de la Crèche (intérieur) renvoie pour l'instant à un unicum. On a le sentiment qu'elle appartient à un noyau plus ancien, d'autant que son axe est-ouest est perpendiculaire à l'orientation du premier bâtiment en bois et ne s'accorde ni avec les orientations de l'église, ni avec celles du bâtiment 1 . Le couloir nord, qui vient chemiser la chapelle de la Crèche, se prolongeait à l'ouest, et, à l'est, la travée droite vient se plaquer contre l'épaulement de l'abside de la chapelle. On peut se demander si le couloir nord (et le sud également) ne succède pas à un système de galeries entourant un plan centré, selon des modèles connus à la même période ${ }^{13}$. Cette idée suggère, par ailleurs, que la crypte actuelle remplacerait un édifice antérieur, dont la présence expliquerait les contraintes reconnues dans la succession des états. Il est bien entendu beaucoup trop tôt pour proposer qu'il puisse s'agir de l'église primitive dédiée à saint Germain d'Auxerre, mais cela doit rester une hypothèse de travail. Il est trop tôt également pour envisager une occupation antérieure à la communauté de Cuxa, dont le bâtiment en bois pourrait 
témoigner, à l'instar des blocs de grand appareil utilisés pour la construction des supports de la nef de l'église abbatiale, dont l'origine antique doit être examinée.

Ces premières observations et les idées qui en découlent, livrées telles quelles, doivent évidemment être renforcées par une approche plus fine des élévations et du sous-sol de la crypte dans le cadre d'une étude qui lui serait consacrée, mais les hypothèses proposées, aussi audacieuses qu'elles puissent paraître, déterminent d'ores et déjà un cadre de recherche et des problématiques.

Malgré les nombreux réaménagements et reconstructions, tant anciens que récents, l'étude menée en 2016 montre à quel point la matière archéologique susceptible d'aider à la compréhension des origines et de l'évolution du site est préservée. En affinant les datations et la succession des états, ce sont les prémices du développement du monastère qui sont aujourd'hui abordées en s'obligeant à un regard nouveau. De même, l'emploi de méthodes nouvelles, du moins sur ce site, comme l'analyse des matériaux du bâti ou les datations radiocarbone, donne d'ores et déjà des résultats prometteurs qui incitent à poursuivre la recherche.

On a déjà eu l'occasion de montrer comment la fouille du cloitre pourrait être particulièrement riche d'enseignements et de découvertes, mais la crypte conserve elle aussi un potentiel précieux, comme nous nous sommes attachés à le montrer ici. L'idéal serait de mener une étude exhaustive de ses élévations avec, au moins dans un premier temps, la fouille - en aire ouverte ou par sondages - du couloir nord et de la chapelle de la Crèche.

Cette fouille devrait permettre de valider, dans le meilleur des cas, les hypothèses d'antériorité, puis de mieux comprendre les relations avec le reste de l'église.

Reçu le : 3 avril 2017 - Accepté le 9 juin 2017

\section{NOTES}

1. P. PONSICH, «Les problèmes de Saint-Michel de Cuxa d'après les textes et les fouilles ", Études roussillonnaises, 2 (1952), p. 21-66.

2. P. PONSICH, "Saint-André d'Eixalada et la naissance de l'abbaye de Saint-Germain de Cuixa (840-879) ", Les cahiers de Saint-Michel de Cuxa, 11 (1980), p. 7-32.

3. P. PONSICH, « Saint-André d'Eixalada... », ibid.

4. P. PONSICH, « Saint-Michel de Cuxa du $\mathrm{IX}^{\mathrm{e}}$ au XII $\mathrm{XI}^{\mathrm{e}}$ siècle. Aperçu historique », Les cahiers de SaintMichel de Cuxa, 1 (1970), p. 20.

5. P. PONSICH, « Saint-Michel de Cuxa... », ibid., p. 20.

6. En raison des décaissements, les niveaux des $\mathrm{X}^{\mathrm{e}}$ et $\mathrm{XI}^{\mathrm{e}}$ siècles apparaissent sur un même plan.

7. A. BERGERET, «L'église Saint-Martin-de-Castries (La Vacquerie-et-Saint-Martin-de-Castries, Hérault), dépendance de l'abbaye de Gellone sur le Larzac ", Archéologie du Midi médiéval, 28 (2010), p. 193-207.

8. Voir par exemple la sépulture 623 de Saint-Nazaire-de-Marissargues à Aubais (Gard), datée par le radiocarbone entre la fin $\mathrm{du} \mathrm{VII}^{\mathrm{e}}$ et le $\mathrm{IX}^{\mathrm{e}}$ siècle [M. OTT, "Saint-Nazaire-de-Marissargues à Aubais (Gard), une église et son cimetière $\mathrm{du} \mathrm{vIII}^{\mathrm{e}}$ au $\mathrm{x}^{\mathrm{e}}$ siècle ", Archéologie du Midi médiéval, 28 
(2010), p. 147-159] ou encore la sépulture 85 de La Granède à Millau (Aveyron), datée, également par le radiocarbone, entre la seconde moitié $\mathrm{du}_{\mathrm{VII}}^{\mathrm{e}}$ et le VIII ${ }^{\mathrm{e}}$ siècle [C. SAINT-PIERRE, « Millau, La Granède (Aveyron) : une église paléochrétienne anonyme sur un éperon barré », Archéologie du Midi médiéval, 28 (2010), p. 181-191].

9. Poz-84916, $1095 \pm 30$ BP.

10. Cette connaissance peut relever d'une tradition établie, d'un marquage au sol, ou avoir été révélée à l'occasion des terrassements pour la construction du mur est.

11. Poz-84919, $1075 \pm 30 \mathrm{BP}$.

12. La galerie pourrait alors desservir un dortoir ou le relier à une église située au sud. A Fontenelle (Saint-Wandrille), les Gestes de l'abbé Anségise donnent le bâtiment ouest comme dortoir au début $\mathrm{du} \mathrm{IX}^{\mathrm{e}}$ siècle, cf. Chronique des abbés de Fontenelle, éd., intro. et trad. fr. PRADIÉ, Paris, 1999, cité par C. SAPIN, «Contribution à l'étude des origines de l'espace claustral de SaintMichel de Cuxa », Les cahiers de Saint-Michel de Cuxa, 46 (2015), p. 229-233.

13. San Miguel de Terrassa (Catalogne), Nea Ekklesia de Basile II (Constantinople).

INDEX

Mots-clés : abbaye, bâtiments monastiques, crypte, sépultures

Index géographique : France/Codalet, Saint-Michel de Cuxa

\section{AUTEUR \\ FABRICE HENRION}

CEM, Auxerre 